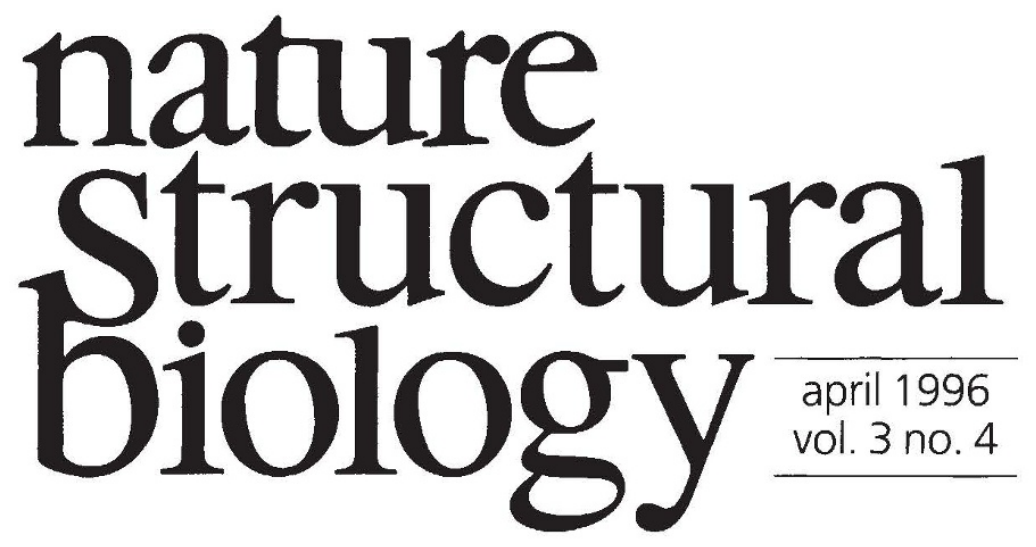

\title{
Between fidelity and promiscuity
}

An enjoyable scientific meeting combines each morsel of research into a continuous blend of scientific progress, with no lumps of excessive contribution from any one area. This is not least because a participant will always be ready for more throughout a lengthy meeting. It is also arguably a model for how a biological problem should be tackled: on all fronts, examining physiology, genetics, enzymology, and structure at the same time. Although no scientific meeting could satisfy this ideal, a recent Keystone Symposium entitled Molecular Mechanisms in DNA Replication and Recombination* made a commendable and mostly successful attempt. Results were reported from a wide range of experimental approaches applied to understanding the fundamental biological problems of replication and recombination, and although no stunning new discoveries were announced, those data that were presented were well integrated, so that the advance in understanding could be appreciated across the whole field.

The common features of replication were presented by J. Hurwitz (Sloan-Kettering Cancer Center, New York) in his keynote talk. Replication is carried out by large complexes; it requires a helicase-primase association and a polymerase-helicase association; it features leading/lagging strand asymmetry; and it has a mechanism of tethering the DNA to polymerase, making it processive. For yeast, Escherichia coli, and phage T4 the tether consists of a circular 'clamp' around the DNA put in place by a clamp loader. More generally, the replication machinery must find and assemble at the correct place on the DNA, at the appropriate time in the cell cycle. Although common features of replication are apparent, a unified picture at the molecular level seems problematic, due to differences in detail between the large numbers of organisms studied.

The three major sub-assemblies of chromosomal DNA polymerase holoenzymes are the DNA polymerase, a sliding clamp, and a clamp loader (in E. coli pol III, all together comprising 10 subunits). Considerable attention at the meeting was given to questions about how the holoenzyme assembles and, in particular, how the clamp is loaded onto the DNA by the clamp loader. Structures of the relevant proteins are informative in this regard. J. Kuriyan (Rockefeller University, New York) reminded us that for $E$. coli $(\beta)$ and yeast (PCNA), the 'clamp' structure is a six-fold symmetric ring that surrounds the DNA dur-

* Molecular Mechanisms in DNA Replication and Recombination, 10-16 February 1996, Taos, New Mexico, USA ing replication (Fig. 1). The gene product (gp) 45 from phage T4 also fits this pattern. These proteins have a net negative charge, but the inner lining of the ring has a positive charge. The T4 clamp does not appear as nicely ring-shaped as the other two, having a slightly thinner, 'squashed' appearance. Consistent 


\section{IMAGE \\ UNAVAILABLE \\ FOR \\ COPYRIGHT \\ REASONS}

Fig. 1 A diagram of conserved residues within the polymerase $\alpha$ active site, summarizing data gathered from sitespecific mutation at key residues. Tyr 865, critical for insertional fidelity, is thought to make direct hydrogen bonds with the incoming base. Figure courtesy of T. Wang.

\section{IMAGE UNAVAILABLE FOR COPYRIGHT REASONS}

Fig. 2 Reconstructions based on electron microscopic data of the T7 hexameric helicase gp4B (top) and the RuvB dodecamer (bottom). Figure courtesy E. Egelman. with this structural impression of flimsiness, in T4 replication the gp 45 clamp falls off DNA quite easily, and is stabilized by gp 55, a promoter recognition protein that in this case acts as a processivity factor-incidentally suggesting a link between replication and transcription (P. Geiduschek, University of California, San Diego). Kuriyan also presented a preview of PCNA bound to a p21-derived peptide. The interaction with p21 suggests a route for cell-cycle mediation of PCNA function.

The mechanism of the clamp loading process is being examined by several groups. In one approach, fluorescent labels are bound to cysteins engineered into specific sites of gp45. Changes in fluorescence can be monitored as the clamp loader binds and ATP is hydrolysed (S. Benkovic, Pennsylvania State University, University Park; P. von Hippel, University of Oregon, Eugene). These studies-along with more biochemical experiments such as the recent work ${ }^{1}$ from the lab of M. O'Donnell showing that the pol III clamp loader and polymerase are in a DNA-mediated competition for an overlapping binding site on the clamp-provide context for a further understanding of how the clamp is loaded onto DNA. The structure of a clamp loader, both alone and complexed with a clamp, is eagerly awaited.

Structural information on DNA polymerases has motivated mutational studies to gain insight into function. For rat DNA polymerase $\beta$ (involved in gap excision repair; unlike some DNA polymerases it lacks a proof-reading mode), three functionally important residues are close enough to interact with DNA through hydrogen bonds to either the template or the incoming nucleotide. In particular, mutations at one of these critical positions to Ala decrease catalytic activity, which is also linked to an increase in the error rate (T. Kunkel, NIEHS, National Institutes of Health, Research Triangle Park, North Carolina). Mutations of specific residues of the human polymerase $\alpha$ (T. Wang, Stanford University School of Medicine, California) can alter specific features of enzyme activity. For example, Tyr 865 probably interacts directly with the base of incoming nucleotides (Fig, 1) and changes in this residue affect insertional fidelity.

There was some discussion about the functional oligomeric state of helicases involved in DNA replication. A reconstruction based on electron microscopy studies of the gp4B helicase from T7 confirms biochemical studies showing that it is a hexamer (Fig. 2; E. Egelman, University of Minnesota, Minneapolis). The hexamer can be assembled on ssDNA, which passes through the central hole, with the small end of the helicase pointing towards the 5' end of the DNA; the helicase unwinds DNA in the $5^{\prime}$ to 3 ' direction. The beginnings of a proposed mechanism for this helicase (S. Patel, Ohio State University, Columbus) are based on the observation that at any given time three weak and three strong dNTP binding sites exist (the hexamer binds three dNTPs and there is considerable negative cooperativity between sites). Only one dNTP hydrolyses at a time. It may be that hydrolysis at successive sites around the circumference of the hexamer drives this helicase forward, the DNA going through the centre and then partly around the inner rim of the molecule.

Not all helicases are hexameric. The Rep helicase of E. coli functions as a dimer (T. Lohman, Washington University, St. Louis, Missouri). Each end of the molecule is a DNA-dependent ATPase. Association of one subunit with ssDNA and ATP results in dissociation of DNA from the other subunit (which may then have a preference for $\mathrm{dsDNA}$ ) through a proposed allosteric mechanism. Successive cycles of binding and release lead to a proposed model in which this helicase rolls along the DNA. It is interesting to speculate how this mechanism 


\section{IMAGE UNAVAILABLE FOR COPYRIGHT REASONS}

Fig. 3 Structure of the UmuD' protein, showing the dimeric organization surface representation of the curvature looking down the two-fold axis. Figure courtesy of T. Peat. may fit with those suggested for the hexameric helicases. Structural studies of either of these helicases will shed light on DNA and dNTP binding, allostery and catalytic mechanism.

Other molecules are involved in setting the stage for replication to begin. For example, the EBNA-1 protein activates replication of the Epstein-Barr virus at the latent origin of replication. The structure of the complex with DNA (L. Frappier, A. Bochkarev, McMaster University, Hamilton, Ontario) is remarkable. Originally, based on the uncomplexed structure of the core DNA binding domain, it seemed that the protein (which binds DNA as a dimer) would bind DNA through two recognition helices. In the structure of the complex, however, flanking regions of the protein were included (they were required to crystallize the complex). In the complex, the flanking region extends a chain that wraps 'under' the DNA and into the minor groove, forming the largest part of the DNA contact surface. The 'recognition helices' appear to be too remote from the DNA to interact with it in the complex, although they may be involved in the initial steps of DNA binding. Several features of the complex hint at the way in which the protein could open up the DNA helix, perhaps to admit other proteins involved in replication.

Some of the proteins used at the initial stage of replication are chaperones, modifying components of replication into an active form. In $\lambda$, a tetrameric complex of the $\mathrm{O}$ protein induces structural changes at ori $\lambda$. The $\mathrm{C}$-terminal portion of $\mathrm{O}$ loads the helicase $\mathrm{B}$ onto ssDNA in association with the chaperone protein $\mathrm{P}$, which then dissociates from the complex (R. Mc Macken, Johns Hopkins University, Baltimore, Maryland). It will be enlightening to see how the $\mathrm{O}$ protein changes the DNA to allow recognition by the $\mathrm{PB}$ complex. Plasmid $\mathrm{P} 1$ replication also requires chaperones, HSP 70 homologues that convert an inactive dimer of the RepA origin binding protein to an active monomer (S. Wickner, NCI, National Institutes of Health, Bethesda, Maryland).

Studies of recombination, as represented at the meeting, spanned general and meiotic recombination, $\mathrm{V}(\mathrm{D}) \mathrm{J}$ recombination, and viral integration. The E. coli RuvB protein, involved in branch migration, bears a structural resemblance to the gp4B helicase-seen in a reconstruction from electron micrograph images of this double hexamer (E. Egelman, Fig. 2) - despite the absence of any extensive sequence homology other than a shared helicase motif. Mu transposition, coordinated by a transposase tetramer, can resolve through replication after strand transfer at a nick (T. Baker, Massachusetts Institute of Technology, Cambridge) - a clear link between replication and recombination. In TN7, the transposition machinery operates at a double-strand break. Alteration of one of the proteins in the complex, so that only nicks rather than breaks are made (as in $\mathrm{Mu}$ ), converts this mechanism into one requiring a replicative step (N. Craig, Johns Hopkins University). In V(D)J recombination, a strand transfer-like reaction carried out by the RAG 1 and RAG 2 proteins results in a closed DNA hairpin structure being formed at the site of a nick, one end of a strand being joined to the other of the same DNA by trans esterification (M. Gellert, NIDDK, National Institutes of Health, Bethesda). This leads to the idea that the mechanism of RAG $1 / 2$ resembles those involved in integration/transposition in TN10, TN7, Mu and HIV-1.

In E. coli, the RecBCD complex and RecA are the central players in recombination. Arguably the most surprising result of the meeting was centred on RecA; several critical properties of this protein, including homologous DNA pairing, can be carried out by a very small fragment of the molecule in isolation (D. CameriniOtero, NIDDK, National Institutes of Health, Bethesda, Maryland). Another surprise is that RecBCD may contain an RNA component whose sequence is 
apparently not in the E. coli chromosome (G. Smith, Fred Hutchinson Cancer Center, Seattle, Washington). However, the evidence for this last finding is apparently the subject of some controversy.

DNA repair was also represented at this meeting, because of its links to replication and in some cases recombination. The UmuD' gene product is involved in SOS mutagenesis in response to DNA damage in E. coli. The structure of the protein (Fig. 2) together with other results, suggests that UmuD' may form filaments, and provides hints as to how it associates with RecA and polymerase to allow replication through damaged regions of DNA, although with a high error rate $(\mathrm{R}$. Woodgate, NICHHD, National Institutes of Health, Bethesda; T. Peat, Columbia University, New York). One result in this area was the description of a newly discovered activity involved in the repair of the results of incomplete action of topoisomerase 1, which makes a 3' covalent bond with DNA at a nick. If a replication fork moves through at this stage, polymerase falls off, resulting in a double-strand break. The new enzyme cleaves the $3^{\prime}$ tyrosine link to topoisomerase $1(\mathrm{H}$. Nash, NIMH, National Institutes of Health, Bethesda).

Some proteins are widely involved in DNA metabolism, and although they play critical roles in replication and recombination, they are not so easily categorized. Two structures of such proteins stand out: the 3D structure of T7 DNA ligase (D. Wigley, A. Doherty, University of Oxford) reveals some interesting parallels to methytransferases in the way it holds the nucleotide to be used in closing the nick relative to the DNA. The crystal structure of integration host factor - a protein with roles in a variety of processes requiring multisubunit protein interactions on DNA - indicates that it bends DNA through $\sim 160^{\circ}$, an ability that would be useful for bringing together remote areas of DNA and associated proteins (P. Rice, NIMH, National Institutes of Health, Bethesda).

One stated purpose of this meeting was to find biological threads that connect replication and recombination. These two processes would appear in some sense to have opposing outcomes: we sometimes think of replication as preserving the DNA, making exact copies (fidelity), while recombination allows admittance of variability (promiscuity). In the case of viral transposition, for example, opposing purposes are clear (though not in the case of meiotic recombination). Recombination and replication are intimately coordinated in the cell, and they share some similar features; but no more so than replication and transcription. Indeed, connections between the latter two processes seemed more in evidence at this meeting than for the former. Perhaps the connection is grounded more in collegial spirit than scientific observation. Nonetheless, the meeting organizers (M. O'Donnell, Cornell University Medical College, New York; S. West, Imperial Cancer Research Fund, Herts, UK) should have every reason to be happy with the results of their efforts. The advance in the field (since the previous Keystone Symposium on this topic) could be seen, according to one participant, in the mechanistic emphasis afforded by the identification and purification of many of the proteins involved. The only shortcoming was that the large number of registrants (more than 450, according to the symposium administrators, although a glance at the 600 seats in the main meeting hall would have indicated that this is an undercount) and speakers (about 100, including workshop participants) necessitated concurrent afternoon sessions, so participants could not attend everything. The word 'symposium' means 'drinking together'; this spirit is hard to maintain in the face of such a demanding schedule, particularly when virtually every presentation is interesting and there is little time to linger over any particular one.

1. Naktinis, V., Turner, J., O' Donnell, M. Cel/ 84, 137-145 (1996) 